\title{
Miocardiopatía diabética: un punto de vista retrospectivo
}

\author{
Rafael Ramírez ${ }^{\mathrm{a}, \mathrm{b}, *}$, Montserrat Durán ${ }^{\mathrm{a}}$ y Javier Márquez ${ }^{\mathrm{a}}$ \\ a Servicio de Medicina Interna, Hospital de Sant Pau i Santa Tecla, Tarragona, España \\ ${ }^{\mathrm{b}}$ Unidad de hipertensión, dislipemias y riesgo vascular, Hospital de Sant Pau i Santa Tecla, Tarragona, España
}

Recibido el 29 de enero de 2017; aceptado el 7 de agosto de 2017

Disponible en Internet el 10 de enero de 2018

\section{PALABRAS CLAVE Insuficiencia \\ cardiaca; \\ Diabetes mellitus; Miocardiopatía diabética}

\section{KEYWORDS}

Heart failure;

Diabetes mellitus;

Diabetic

cardiomyopathy

\begin{abstract}
Resumen
Objetivo: Estimar la prevalencia de pacientes con probable miocardiopatía diabética (sin exigir biopsia endomiocárdica), analizando sus características y comparándolas con pacientes no diabéticos con insuficiencia cardiaca idiopática.

Métodos: Estudio retrospectivo sobre una cohorte de 270 pacientes que ingresaron de manera consecutiva por insuficiencia cardiaca. De estos se excluyeron del estudio todos aquellos que tuvieran una causa conocida de insuficiencia cardiaca (cardiopatía isquémica, hipertensiva, hipertrófica, valvular u otras). En total 58 pacientes, 18 de los cuales eran diabéticos (la causa de la insuficiencia cardiaca pudo ser la miocardiopatía diabética) y 40 sin diabetes (insuficiencia cardiaca idiopática). Se procedió a comparar las características entre ambos grupos. El análisis estadístico y la comparación de las distintas variables se realizó mediante el programa SPSS versión 20.0 .

Resultados: De 270 pacientes estudiados, 18 podrían haber sido diagnosticados con miocardiopatía diabética, lo que supone hasta un $6 \%$ de la insuficiencia cardiaca en esta serie.

Conclusiones: Se considera que este estudio puede hacer reflexionar sobre la prevalencia global de la miocardiopatía diabética, sus características y sus dificultades diagnósticas.

(c) 2017 Sociedad Colombiana de Cardiología y Cirugía Cardiovascular. Publicado por Elsevier España, S.L.U. Este es un artículo Open Access bajo la licencia CC BY-NC-ND (http:// creativecommons.org/licenses/by-nc-nd/4.0/).
\end{abstract}

\section{Diabetic cardiomyopathy: a retrospective point of view}

\section{Abstract}

Objective: To determine the prevalence of patients with probable diabetic cardiomyopathy (without requesting an endomyocardial biopsy), as well as to analyse its characteristics and comparing them with non-diabetic patients with idiopathic heart failure.

\footnotetext{
* Autor para correspondencia.

Correo electrónico: rramirez@xarxatecla.cat (R. Ramírez).
} 
Methods: A retrospective study was conducted on a cohort of 270 patients consecutively admitted to hospital due to heart failure. Excluded from the study were those who had a known cause of heart failure (ischaemic, hypertensive, hypertrophic, valvular, or other causes of heart disease). This left a total of 58 patients, 18 of whom were diabetics (the cause of heart failure could be due to diabetic cardiomyopathy) and 40 non-diabetics (idiopathic heart failure). A comparison was made between the characteristics of both groups. The statistical analysis and the comparison of the different variables were performed using Version 20.0 of the SPSS program.

Results: Of the 270 patients studied, 18 could have been diagnosed with diabetic cardiomyopathy, which would be $6 \%$ of the cardiac failures in this series.

Conclusions: It is believed that this study can lead to reflecting on the overall prevalence of diabetic cardiomyopathy, its characteristics, and the difficulties in its diagnosis.

(c) 2017 Sociedad Colombiana de Cardiología y Cirugía Cardiovascular. Published by Elsevier España, S.L.U. This is an open access article under the CC BY-NC-ND license (http:// creativecommons.org/licenses/by-nc-nd/4.0/).

\section{Introducción}

El concepto de disfunción miocárdica directamente producida por la diabetes mellitus fue sugerido por Lundbaek en $1969^{1}$, tras observar durante años que hasta dos tercios de los ancianos con diabetes mellitus la presentaban². No obstante, fue hasta 1974 cuando Rubler describió un nuevo tipo de cardiomiopatía asociada a la diabetes mellitus, a partir de las necropsias de cuatro pacientes diabéticos con insuficiencia cardíaca congestiva y arterias coronarias sanas $^{3}$. Desde entonces se definió la miocardiopatía diabética como la disfunción ventricular que ocurre en los pacientes diabéticos con independencia de la cardiopatía isquémica o de la hipertensión ${ }^{4}$. Posteriormente, se ha añadido la necesidad de excluir también la cardiopatía valvular, la cardiopatía congénita y la miocardiopatía viral o infiltrativa para diagnosticar la micoardiopatía diabética. Para ello se requiere la realización de biopsia endomiocárdica ${ }^{5}$, por lo que ante lo invasivo de la misma y su limitada disponibilidad, se dificulta el diagnóstico de miocardiopatía diabética. Por otra parte, a pesar de ser bien conocido que gran parte de la morbilidad y mortalidad de la diabetes mellitus se debe a las enfermedades cardiovasculares, clásicamente tan sólo ha sido considerada como un factor de mal pronóstico por las guías de práctica clínica para el manejo de la insuficiencia cardiaca, que no han contemplado la existencia de la miocardiopatía diabética como una entidad etiológica específica6 ${ }^{6}$.

\section{Métodos}

El objetivo de esta observación clínica fue tratar de estimar la prevalencia de pacientes que podrían cumplir con el diagnóstico de miocardiopatía diabética (sin exigir la biopsia endomiocárdica) en un grupo de pacientes con insuficiencia cardiaca sin causa conocida, analizando sus características y comparándolas con pacientes no diabéticos del mismo grupo.
Para ello, sobre una serie de 270 pacientes (128 de los cuales eran diabéticos) que ingresaron consecutivamente en nuestro centro por insuficiencia cardiaca, se estudió de manera retrospectiva la posible presencia de miocardiopatía diabética tras excluir aquellos con causas conocidas de insuficiencia cardiaca: cardiopatía isquémica (un total de 124 pacientes), miocardiopatía hipertensiva/hipertrófica (62 pacientes de los 270 iniciales), valvular, congénita u otras etiologías conocidas (26 pacientes en total). En aquellos con más de un ingreso durante el periodo de estudio, sólo se consideró el primero de ellos. Finalmente, quedaron 58 pacientes con insuficiencia cardiaca sin causa conocida que fueron divididos en dos grupos: uno conformado por 18 pacientes con insuficiencia cardiaca sin causa conocida y diabetes (y que por tanto podrían tener miocardiopatía diabética) y otro con 40 no diabéticos con insuficiencia cardiaca idiopática. Las variables cuantitativas se expresaron como media \pm desviación estándar y se compararon mediante la prueba de la t de Student o ANOVA. Se utilizó la prueba de

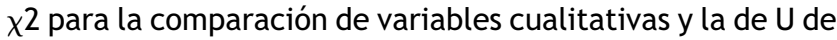
Mann-Whitney para datos no paramétricos. Para el análisis estadístico se empleó el programa SPSS en su versión 20.0. Se consideró significativo un valor de $p<0,05$.

\section{Resultados}

De 270 insuficiencias cardiacas estudiadas, hasta 18 de ellas podrían atribuirse a miocardiopatía diabética, lo que supone hasta un $6 \%$ de las insuficiencias cardiacas de esta serie.

Los resultados de la comparación pueden observarse en la tabla 1.

\section{Discusión}

Todos los casos de miocardiopatía diabética se trataron de pacientes con diabetes mellitus tipo 2 y con un tiempo de diagnóstico de la diabetes superior a cinco años, tal como los descritos por Rubler ${ }^{3}$. Esta estimación debe ser puesta en perspectiva teniendo en consideración que la prevalencia 
Tabla 1 Comparación de los pacientes con insuficiencia cardiaca debida a probable miocardiopatía diabética y aquellos con insuficiencia cardiaca idiopática sin diabetes

\begin{tabular}{|c|c|c|c|}
\hline & $\begin{array}{l}\text { Probable miocardiopatía } \\
\text { diabética }(n=18)\end{array}$ & $\begin{array}{l}\text { Pacientes no diabéticos con } \\
\text { insuficiencia cardiaca } \\
\text { idiopática }(n=40)\end{array}$ & $\mathrm{P}$ \\
\hline Edad años media (DE) & $76(5)$ & $74(10)$ & 0,35 \\
\hline Sexo (varón) (\%) & 39 & 60 & 0,16 \\
\hline HTA (\%) & 72 & 37 & 0,02 \\
\hline DLP (\%) & 50 & 12 & 0,00 \\
\hline Tabaquismo (\%) & 17 & 21 & 0,88 \\
\hline NYHA II-III (\%) & 72 & 71 & 0,75 \\
\hline Fibrilación auricular (\%) & 56 & 72 & 0,23 \\
\hline Fracción eyección (\%) media (DE) & $51(15)$ & 50 (17) & 0,86 \\
\hline \multicolumn{4}{|l|}{ Tratamiento (\%) } \\
\hline Betabloqueante & 33 & 30 & 0,8 \\
\hline IECA o ARA2 & 61 & 57 & 0,42 \\
\hline Anticoagulantes & 39 & 33 & 0,63 \\
\hline AAS & 50 & 15 & 0,01 \\
\hline Estatinas & 44 & 8 & 0,02 \\
\hline \multicolumn{4}{|l|}{ Resultados de laboratorio } \\
\hline Glucosa (mg/dl) & $149(38)$ & $100(15)$ & 0,00 \\
\hline $\mathrm{Hb}$ A1c $(\%)$ & $8,6(2,3)$ & $6,1(0,6)$ & 0,00 \\
\hline Colesterol total $(\mathrm{mg} / \mathrm{dl})$ & $162(37)$ & $154(40)$ & 0,47 \\
\hline Colesterol LDL (mg/dl) & $82(15)$ & $99(29)$ & 0,31 \\
\hline Colesterol HDL (mg/dl) & $42(10)$ & $42(10)$ & 0,98 \\
\hline Triglicéridos (mg/dl) & $134(59)$ & $103(38)$ & 0,02 \\
\hline Urea $(\mathrm{mg} / \mathrm{dl})$ & $49(22,2)$ & 62,5 (39) & 0,11 \\
\hline Creatinina $(\mathrm{mg} / \mathrm{dl})$ & $1,1(0,3)$ & $1,3(0,7)$ & 0,06 \\
\hline Filtrado glomerular $\left(\mathrm{ml} / \mathrm{min} / \mathrm{m}^{2}\right)$ & $68(37)$ & $56(20)$ & 0,11 \\
\hline GOT (UI/L) & $24(10)$ & 35 (39) & 0,08 \\
\hline GPT (UI/L) & $26(20)$ & $44(61)$ & 0,11 \\
\hline Hemoglobina (g/dl) & $11,8(2,3)$ & $12,1(2,7)$ & 0,73 \\
\hline Albúmina $(\mathrm{g} / \mathrm{dl})$ & $3,5(0,4)$ & $3,4(0,5)$ & 0,46 \\
\hline Ácido úrico (mg/dl) & $6,9(2,9)$ & $7,6(2,8)$ & 0,42 \\
\hline
\end{tabular}

de insuficiencia cardiaca es de un 1 a $2 \%$ de la población en los países desarrollados ${ }^{7}$, y que a nivel mundial más de 380 millones de personas padecen diabetes mellitus, con expectativa de incrementarse hasta unos 592 millones en el año $2035^{8}$.

No existieron diferencias significativas en cuanto a edad, sexo, tabaquismo, estadio de la NYHA, fracción de eyección o presencia de fibrilación auricular entre los pacientes con posible miocardiopatía diabética y aquellos con insuficiencia cardiaca idiopática sin diabetes. Los pacientes con posible miocardiopatía diabética como causa de la insuficiencia cardiaca tuvieron más prevalencia de hipertensión arterial y dislipidemia, de acuerdo con la tendencia conocida a la agregación que factores de riesgo cardiovascular clásicos se suman a los pacientes con diabetes mellitus ${ }^{9}$; en consecuencia, recibían con mayor frecuencia tratamiento con ácido acetil salicílico y estatinas. En cambio, no existieron diferencias en el tratamiento con inhibidores de la enzima convertidora de angiotensina o antagonistas de los receptores de angiotensina 2, betabloqueantes o anticoagulantes entre ambos grupos. Los pacientes con posible miocardiopatía diabética presentaron significativamente mayores cifras de glucosa en ayunas, hemoglobina glicosilada y triglicéridos. Estos resultados concuerdan con dos de los principales mecanismos fisiopatológicos descritos para la miocardiopatía diabética; la hiperglicemia vía acumulación y activación de AGE (advanced glycated end-products) y de ROS (reactive oxigen species) miocárdica contribuye a la fibrosis y rigidez ventricular mientras que la lipotoxicidad de los triglicéridos y los ácidos grasos libres genera apoptosis de cardiomiocitos $^{5,9}$.

Pese a que las guías de práctica clínica para el manejo de la diabetes mellitus, la prediabetes y las enfermedades cardiovasculares de la Sociedad Europea de Cardiología (ESC) en colaboración con la Sociedad Europea para el Estudio de la Diabetes caracterizan bien la miocardiopatía diabética ${ }^{9}$, las recientemente publicadas guías de práctica clínica para el manejo de la insuficiencia cardiaca de la propia Sociedad Europea de Cardiología, siguen sin contemplar la existencia de la miocardiopatía diabética como una entidad específica ${ }^{7}$. En cualquier caso, debe valorarse como un avance que, además de contemplar a la diabetes mellitus como una comorbilidad, la incluyen ya en la tabla de los agentes causales de insuficiencia cardiaca ${ }^{7}$, dejando de 
considerarla como un mero factor de mal pronóstico tal como hacían las anteriores guías ${ }^{6}$. En este sentido, para progresar en su reconocimiento sería deseable poder alcanzar el diagnóstico de miocardiopatía diabética más fácilmente sin necesidad de tener que recurrir sistemáticamente a la biopsia endomiocárdica. Es posible que la implantación de nuevas técnicas de imagen como la resonancia magnética cardíaca, con reconocida capacidad en el diagnóstico etiológico de la insuficiencia cardiaca ${ }^{7,10}$ pueda ser útil para ello. Así mismo, existen técnicas como la ecocardiografía doppler tisular en reposo y esfuerzo así como los niveles de péptido natriurético procerebral $\mathrm{N}$ terminal (NT-proBNP) que pueden detectar disfunción ventricular incipiente en pacientes diabéticos asintomáticos y que podrían facilitar un diagnóstico precoz de esta entidad si bien en la revisión bibliográfica realizada se encuentran artículos en los que se emplean dichas técnicas y marcadores en pacientes diabéticos con y sin cardiopatía isquémica ${ }^{11}$.

\section{Conclusiones}

Esta observación ha de ser interpretada con precaución ante el reducido número de pacientes analizados y su carácter retrospectivo. Esto último ha impedido recoger variables de resistencia a la insulina, cuya existencia se reconoce como uno de los mecanismos fisiopatológicos de la miocardiopatía diabética que disminuye la contractilidad miocárdica ${ }^{5,9}$. No obstante, la ausencia de biopsia endomiocárdica supone la principal limitación, pues pudiera haber hecho sobreestimar la posible presencia de miocardiopatía diabética. En cualquier caso, se considera que este trabajo puede hacer reflexionar sobre las características de la miocardiopatía diabética, las dificultades para su diagnóstico en la actualidad y la prevalencia global que podría generar esta entidad.

\section{Conflicto de intereses}

Ninguno.

\section{Financiación}

Ninguna.

\section{Bibliografía}

1. Lundbaek K. Is there a diabetic cardiopathy? En: Schettler G, editor. Pathogenetische faktoren des myokardinfarkts'. Stuttgart: Schattauer; 1969. p. 63-71.

2. Lundbaek K. Diabetic angiopathy. A specific vascular disease. Lancet. 1954;263:377-9.

3. Rubler S, Dlugash J, Yuceoglu YZ, KumralT, Branwood AW, Grishman A. New type of cardiomyopathy associated with diabetic glomerulosclerosis. Am J Cardiol. 1972;30:595-602.

4. Boudina S, Abel ED. Diabetic cardiomyopathy revisited. Circulation. 2007;115:3213-23.

5. Seferovic PM, Paulus WJ. Clinical diabetic cardiomyopathy: a two-faced disease with restrictive and dilated phenotypes. Eur Heart J. 2015;36:1718-27.

6. McMurray JJ, Adamopoulos S, Anker SD, Auricchio A, Bohm M, Dickstein $\mathrm{K}$, et al. ESC guidelines for the diagnosis and treatment of acute and chronic heart failure 2012. Eur J Heart Fail. 2012;14:803-69.

7. Ponikowski P, Voors AA, Anker SD, Bueno H, Cleland JG, Coats AJ, et al. 2016 ESC Guidelines for the diagnosis and treatment of acute and chronic heart failure. Eur J Heart Fail. 2016;18:891-975.

8. Forouhi NG, Wareham NJ. Epidemiology of diabetes. Medicine. 2014;42:698-702.

9. Ryden L, Grant PJ, Anker SD, Berne C, Cosentino F, Danchin $\mathrm{N}$, et al., collaboration with the EASD. ESC Guidelines on diabetes, pre-diabetes, and cardiovascular diseases developed in. Eur Heart J. 2013;34:3035-87.

10. Gonzalez JA, Kramer CM. Role of imaging techniques for diagnosis, prognosis and management of heart failure patients: cardiac magnetic resonance. Curr Heart Fail Rep. 2015;12: 276-83.

11. Castaño Rodríguez S, Coma-Canella I, López Salazar B, Barba Cosials J. Echocardiographic findings and NT-proBNP level in type-2 diabetic patients with and without ischemic heart disease. Rev Esp Cardiol. 2009;62:1184-8. 